\title{
GAIA Level 3 Maternal Immunization
}

National Cancer Institute

\section{Source}

National Cancer Institute. GAIA Level 3 Maternal Immunization. NCI Thesaurus. Code C127983.

GAIA Level 3 Maternal immunization is defined by two criteria: first, the woman/medical attendant reports the pregnancy; second, the woman reports receipt of vaccination during pregnancy, but no formal recording of the immunization is available. 